
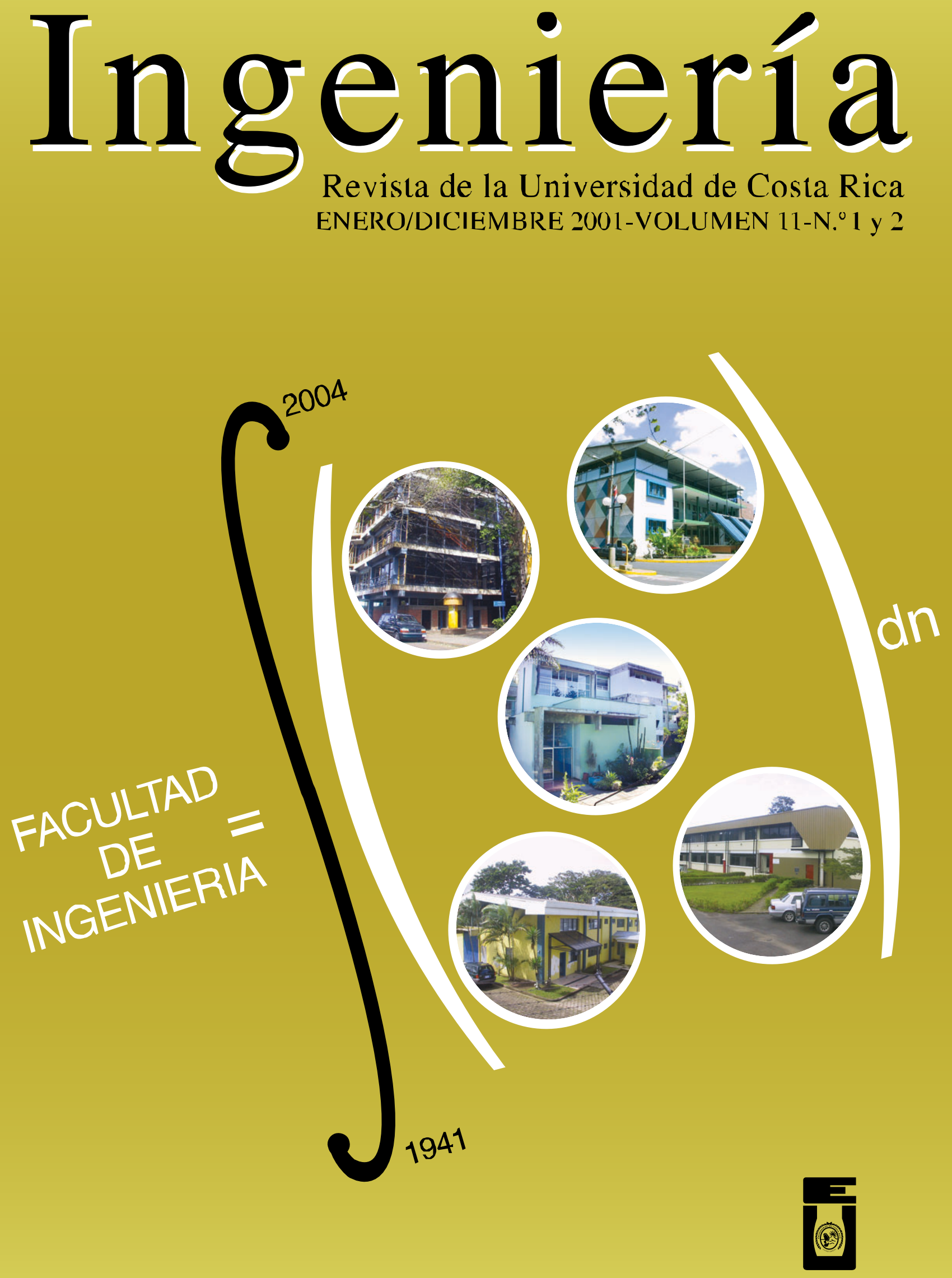


\title{
MEASURING CUTTING FORCES IN MACHINING PROCESSES
}

\author{
Horacio Vásquez Céspedes, M.Sc.
}

\begin{abstract}
The principle of operation of force sensors most commonly used in machining processes and the trends of this technology are presented in this paper. Force sensors are needed because measuring cutting forces during machining processes is the most important and basic step to determine and control the conditions under which the machine, the tool, and the workpiece are performing. In the majority of applications the measurement of cutting forces during machining is done using dynamometers based on either strain gages or piezo-electric transducer with quartz sensors. Recently, micro-electro-mechanical systems (MEMS) are being designed and constructed using polysilicon beam resonators to measure forces. These sensors relate the force that is being measured to the frequency of vibration of the resonator. In recent research projects and studies, there is a tendency to transfer the force sensor signals by wireless technology.
\end{abstract}

\section{INTRODUCTION}

Measuring cutting forces during machining processes is the most important and basic step to determine and control the conditions under which the machine, the tool, and the workpiece are performing. Through measuring cutting forces, geometry of the tool, and analysis of the chip formation that takes place during machining, it is possible to estimate the operating temperatures, tool wear, and power consumption, among other properties that are important to improve the process. On-line computation of these parameters is required to estimate and control the quality of the finished product as well as to consider maintenance actions for the optimal operation of the machine and the tool. In general, adequately designed and implemented control systems are incorporated to improve and optimize the machine tool cutting processes. Force transducers, or sensors, that generate the inputs needed for the decision-making strategy of the control system, are always among the most important parts of the equipment required to achieve this task. This paper describes the force sensors most commonly used during machining processes and presents the trends of the technology in this field.

\section{FORCE SENSORS}

\subsection{IMPORTANCE OF FORCE SENSORS IN MACHINING PROCESSES}

Force sensors are the best alternatives for monitoring tool wear, and to estimate power consumption, temperatures and other important parameters during a cutting process [10]. Force is a physical quantity that traditionally could be obtained indirectly from any of the following physical quantities:

- $\quad$ Displacement: $\mathrm{F}=\mathrm{k} \Delta \mathrm{x}$; where $\mathrm{k}$ is either a spring constant, gage factor or other similar constant

- Acceleration: $\mathrm{F}=\mathrm{m}$ a; where $\mathrm{m}$ is the mass

Pressure: $\mathrm{F}=\mathrm{PA}$; where $\mathrm{A}$ is the area.

\footnotetext{
* Profesor Asociado, Ingeniería Mecánica, Universidad de
} Costa Rica 
Consequently, many force transducers are based on detection of any of these three quantities. A strain sensor could be seen as a sensor that converts changes of displacement to changes in an electrical property such as resistance or voltage; therefore, strain sensors could also be considered displacement sensors.

Some requirements for a force sensor used to measure cutting forces in machining are rigidity, sensitivity, and accuracy [2]. Force sensors must be rigid enough to keep the tool in place, and at the same time, they must be flexible to be capable of indicating deformation. The accuracy of force sensors must be within $\pm 1 \%$ of the reading, and the sensitivity must be one pound increments, or less. The static stiffness required for the sensor must be of the order of $10^{8} \mathrm{~N} / \mathrm{m}$, and the criterion to be used is that the natural frequency of the force sensor must be at least 4 times the frequency of the exciting vibrations in the particular applications [1]. For example, if a turning process is done at 1 $000 \mathrm{rpm}$, which is equivalent to an excitation frequency of $107 \mathrm{rad} / \mathrm{s}$, then the dynamometer must have a natural frequency of at least $427 \mathrm{rad} / \mathrm{s}$. Since the natural frequency of the dynamometer depends on its stiffness and mass, either the stiffness could be increased or the mass decreased in order to obtain higher natural frequency.

Another important requirement for a force sensor in machining application is the no existence of cross sensitivity. In general the sensor must measure at least two perpendicular forces, for example $\mathrm{F}_{\mathrm{P}}$ and $\mathrm{F}_{\mathrm{Q}}$, and it is desirable that the sensing devices that detect the force $F_{\mathbf{P}}$ be as insensitive as possible to force $\mathrm{F}_{\mathrm{Q}}$, and vice versa.

Besides, force sensors, or force transducers, must be stable with respect to time, temperature, and humidity, and specific properties such as size, ruggedness, and adaptability are essential for particular applications. It is important that the transducer be linearly calibrated to avoid the need of a single reference point to express the measured forces [1]. In machining processes, only force sensors capable of measuring deflections of about $0,025 \mathrm{~mm}$ to $1 \mathrm{~mm}$, or less, are of interest to execute the measurement, having at the same time the stiffness required [1]. In addition, force sensors has to be protected to avoid contact with cutting fluids, chips, or any other possible cause of damage during cutting.

Some ways to control cutting processes include predictors based on neural networks or other kind of artificial intelligence devices or algorithms whose feedback and statistical database are obtained in part from force sensors.

\subsection{FORCE SENSORS BASED ON BONDED STRAIN GAGES}

A strain gage is a small electrical device consisting of a long wire wound as a flat coil, and the most common ones have a nominal electrical resistance value of $120 \Omega$ or $350 \Omega$. The coil is built between thin insulating plastic layers, and to assure electrical isolation between the gage wire and the metal, to which the gage is installed, at least $50 \mathrm{M} \Omega$ of electrical resistance must prevail after installation. The gage is installed on the metal surface and at the point and direction where the strain is to be measured. Strain gages are based on the electrical resistance properties of wires with uniform cross sectional area, A, as presented in Eq. (1):

$$
R=\frac{\rho L}{A}
$$

where $\rho$ is the resistivity of the material, $L$ is the length, and A the cross sectional area of the wire. From Eq. (1), it is determined that when the length of the strain gage wire changes, its resistance also changes, and this effect is the most important one to measure strain. Foil strain gages are made by etching a constantan foil. Strain gages to measure strain in perpendicular or multiple directions are usually built in the same package.

An important property of strain gages, given by the manufacturer, is the gage factor, GF, which is the sensitivity of the gage, and is equal to the ratio of the change in electrical resistance to the change in length, or strain:

$$
G F=\frac{\Delta R / R}{\Delta L / L}=\frac{\Delta R / R}{\varepsilon}
$$


where $\varepsilon$ is the strain, $\mathrm{L}$ is the wire length, and $\mathrm{R}$ is the nominal value of the electrical resistance of the strain gage. Hence, the strain could be computed with Eq. (2) once the change in the gage's electrical resistance, $\Delta \mathrm{R}$, is measured.

A Wheatstone bridge converts this change in resistance to a change in voltage, and the procedure to do so is explained following.

\subsubsection{WHEATSTONE BRIDGE}

An electric circuit called Wheatstone bridge is shown in figure 1 . This bridge consists of four main resistors: $\mathrm{R}, \mathrm{R}_{1}, \mathrm{R}_{2}, \mathrm{y} \mathrm{R}_{3}$; arranged as shown, in a way such that by measuring the output voltage, $\mathrm{V}_{\mathrm{O}}$, and by knowing the input voltage $V_{i}$, electrical resistance changes, $\Delta R$, in the strain gage could be estimated. When only one strain gage is used a quarter bridge is formed. The resistor $\mathrm{R}_{4}$ and the potentiometer $\mathrm{R}_{\mathrm{t}}$ are used to balance the circuit. This is, the output voltage, $\mathrm{V}_{\mathrm{o}}$, could be adjusted to be zero when the electrical resistance of the strain gage is the nominal value, $\mathrm{R}$, which is the same as when $\Delta R=0$. In general, the output voltage, $\mathrm{V}_{\mathrm{o}}$, is not zero, even when there is not deformation of the strain gage, because the resistors used to complete the bridge are not always identical, and that is the reason why an adjusting zero circuit is necessary.

The input voltage, $\mathrm{Vi}$, must be low, approximately 5 volts, to reduce heat generation in the resistors and strain gages [1].

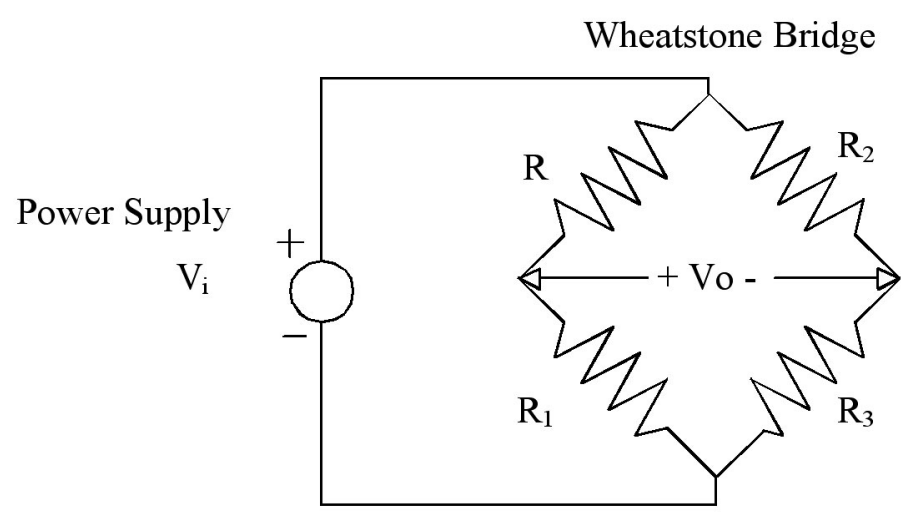

Figure 1. Wheatstone bridge.

\subsubsection{PRACTICAL EXAMPLES OF STRAIN GAGES DYNAMOMETERS}

Example 1: The objective of this example is to obtain the force P, shown in Figure 2, by using two strain gages installed on a rectangular beam. Having always in mind that this beam could be either the tool holder, or the tool shank, and the point of action of the force $\mathrm{P}$ is the cutting edge or tip of the tool. The two strain gages are mounted on the top surface of the cantilever beam as shown in the figure, and are in tension when a load $\mathrm{P}$ is applied to the end of the beam. 


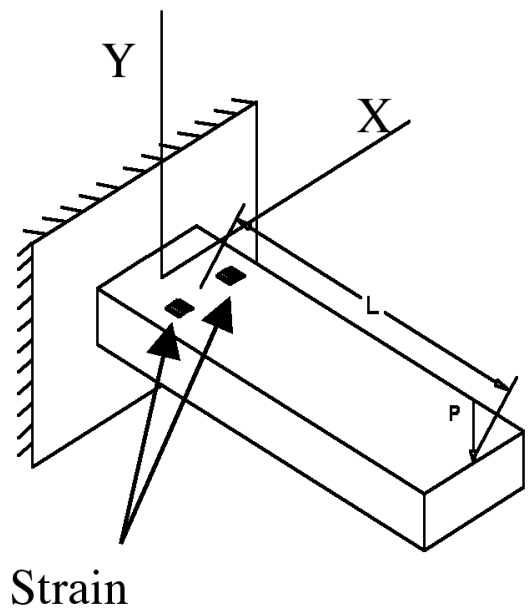

Figure 2. Cantilever beam force sensor.

These strain gages must be connected to a Wheatstone bridge in the positions $\mathrm{R}$ and $\mathrm{R} 2$, or $\mathrm{R} 1$ and R3, as shown in Figure 1, and the other two resistors must be added to complete the bridge, and must have the same nominal value of resistance as the strain gages, so that the bridge be as balance as possible $\left(V_{\mathrm{O}}=0\right)$ when the gages are undeformed.

This configuration is called half bridge and Eq. (3) presents the relationship between the output and input voltages.

$$
\frac{V_{o}}{V_{i}}=\frac{1}{2} \frac{\Delta R}{R}
$$

Therefore, by measuring the output voltage, Vo, the change in electrical resistance can be calculated with Eq. (3), and the strain can be obtained using Eq. (2). The stress on the surface of the beam at the points where the strain gages are installed is given by Eq. (4).

$\gamma=E \varepsilon$

where $\mathrm{g}$ is the stress and $\mathrm{E}$ is Young's modulus of the beam. In this particular example, knowing that the theoretical stress due to load $\mathrm{P}$ at the points where the gages are installed is given by

$\gamma=\frac{6 P L}{a b^{2}}$

where "a" is the width, "b" the thickness of the rectangular beam, and $L$ the distance between the gages and the point of application of load $\mathrm{P}$. From Eqns. (4) and (5) the load P is obtained. Knowing all this relationships among the different parameters, a practical thing to do is to obtain a calibration table, fitting curve, or a plot of force $P$ against output voltage, $V_{O}$, and use either one directly to determine the force $\mathrm{P}$.

To obtain more accurate measurements of force $\mathrm{P}$, it is necessary to install two more strain gages on the bottom surface of the beam and directly below the first two strain gages. These two "new" strain gages will be in compression and under the same magnitude of strain as the other two gages when a force $\mathrm{P}$ is applied. As mentioned before, the gages that are in tension must be located in either positions $\mathrm{R}$ and $\mathrm{R} 2$, or R1 and R3, in the Wheatstone bridge, and this time 
the gages that are in compression must be located in whichever two places are not used by the gages in tension. This configuration is called a full bridge and the following relationship between output and input voltages applies,

$$
\frac{V_{o}}{V_{i}}=\frac{\Delta R}{R}
$$

It is important to notice that forces $\mathrm{Q}$ and $\mathrm{R}$, both perpendicular to force $\mathrm{P}$, also $\mathrm{Q}$ perpendicular to the beam's longitudinal axis, and $\mathrm{R}$ along the longitudinal axis, have negligible effect on the bridge output voltage, which is known as a property of no cross sensitivity or no cross talk [1]. A similar situation occurs with the temperature effect, which could be neglected when all the strain gages are considered to suffer the same temperature changes. This is meaningful because with the installation of another set of strain gages on the lateral walls of the beam, it is possible to measure force $\mathrm{Q}$. Thus, even under conditions of non-zero force $\mathrm{Q}$, and also under a third force component, $\mathrm{R}$, acting at the end of the beam along the beam's longitudinal axis, the full bridge's Eq. (6) is still valid.

Example 2: $\quad$ Strain gages installed on a rectangular cross sectional area of a tool holder as shown in Figure 3, are subject to strains due to forces $\mathrm{P}, \mathrm{Q}$, and $\mathrm{R}$, and due to moments $\mathrm{Mp}$, and $\mathrm{Mq}$ due to the corresponding forces $\mathrm{P}$ and $\mathrm{Q}$.
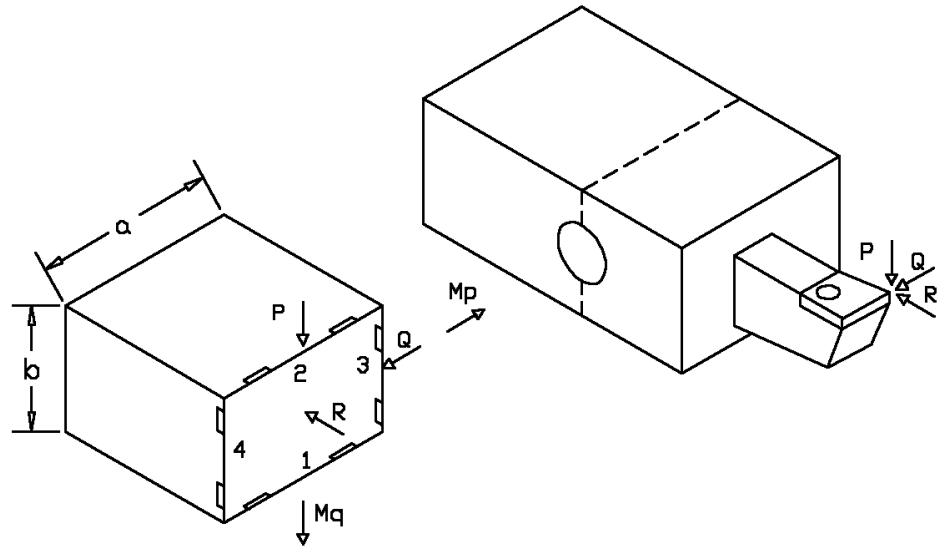

Figure 3. Strain gage dynamometer

A hole has been drilled at the center of the cross sectional area, where the strain gages are installed, to increase the sensitivity of the dynamometer while keeping the tool holder stiffness almost unchanged.

Considering the following conditions for this example:

a) strain gages with a nominal resistance value of $\mathrm{R}$,

b) strain gages of sections 1 and 2 (bottom and top) form a full bridge with output voltage $\mathrm{V}_{\mathrm{O} 1}$,

c) strain gages of sections 3 and 4 (lateral sides) form another full bridge with output
$\mathrm{V}_{\mathrm{O} 2}$; then, Eqns. (7) present the relationship between both Wheatstone bridges' output voltages and the bending moments due to forces $\mathrm{P}$ and $\mathrm{Q}$; but, for simplicity of this explanation, without taking into account the effect of the hole drilled to the beam.

$$
\begin{aligned}
& V_{01}=G \cdot F \cdot \frac{6 M_{P}}{E a b^{2}} V_{i} \\
& V_{02}=G \cdot F \cdot \frac{6 M_{Q}}{E b a^{2}} V_{i}
\end{aligned}
$$


where $V_{i}$ is the input voltage to the bridges, and G.F. is the gage factor of the strain gages.

Notice that there is no cross sensitivity and that force $\mathrm{R}$ does not contribute to these results because it affects in an equal way all the strain gages; consequently, canceling its effect. A similar situation occurs with the temperature effect.

Taking into account that the tool holder is made of a good heat conducting material such as aluminum, then it is a reasonable assumption that the temperature of all the strain gages is about the same. As a result, changes in temperature affect all the strain gages equally, canceling it effect on the final measurement. To measure the axial force $\mathrm{R}$, additional strain gages must be installed.

Example 3: $\quad$ Reference [4] presents the first prototype of a dynamometer that was integrated within the tool shank with the objective of measuring cutting forces during turning. This dynamometer had also as requirements to be easy to install, use, and transmit data through wireless equipment [4]. In addition, other purposes to build this dynamometer were to study the following aspects:

\begin{tabular}{ll}
- & Tool wear \\
- & Tool breakage \\
- & Surface quality \\
- & Cutting temperatures \\
\hline
\end{tabular}

Strain gages were selected as the sensor integrated within the tool shank because of the following reasons:

- $\quad$ Sensitivity that allows a stress to be detected while keeping good stiffness

- $\quad$ Direct application to the tool with minimum modification of shape and size.

- $\quad$ Relatively simple signal conditioning

A location relatively far from the tool tip was selected to avoid thermal transients and to increase sensitivity, but temperature compensation was necessary anyway [4]. Usually strain gages are installed near a hole or a high concentration stress region to amplify the strain, but without reducing the stiffness of the tool. In this particular application done by Santochi and others, the voltage obtained from the Wheatstone bridge was amplified 200 times, and later the amplified signal was converted from voltage to frequency by an integrated circuit, and sent to an infra-red emitter diode placed in the rear side of the tool. For an optical signal to be transmitted adequately, the photodiode receiver must be on the axis of the emitter diode. In the receiver, the signal was amplified again, and sent to a frequency to voltage converter [4]. The final signal that was obtained was a combination of a voltage proportional to the strain, and an offset voltage due to unbalanced bridge, thermal effect, etc. However, by measuring the signal when the tool is not cutting the offset component could be eliminated. Figure 4 shows the basic idea of the procedure followed during this experiment. 


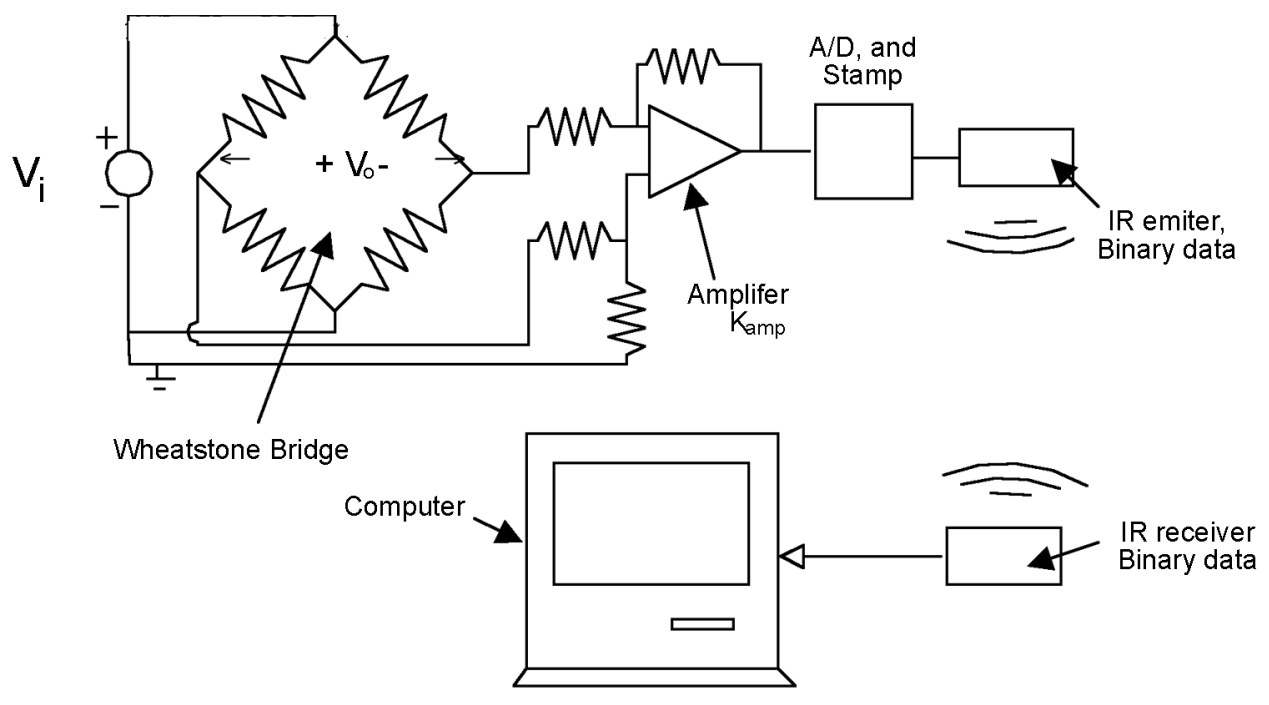

Figure 4. Strain gage dynamometer with wireless transmission of the signal

The signal processing circuitry was in charge of amplifying and transmitting the signal, and of receiving and decoding the signal on the other side. The first part of the circuitry was within the tool shank, while the second part was away from the machine but on the axis direction of the infrared emitter. After some cutting was done the results were compared with the ones obtained with a piezo-electric dynamometer, and positive results were obtained. The cost of the system was acceptable, the adaptability to workshop conditions was good, the installation was quick and easy, the tool did not required any modification, and the change of stiffness was negligible [4]. It seems (not mentioned in the paper) that for this experiment only four strain gages were installed in a strategic position of the tool shank and connected to a Wheatstone bridge. The most impor- tant result obtained was that the output of the integrated sensor was proportional to a linear combination of the three force components measured with the piezo-electric dynamometer, during both a roughing and a finishing operation. It was determined that the effect of the power force, P, was $69 \%$, of the feed force, Q, was $23 \%$, and of the back force, R, was $8 \%$, on the sensor's output voltage [4]. With this first prototype of sensor integrated tool, it was demonstrated that a well-calibrated single output signal might be enough to acquire the three components of the cutting forces during turning [4]. Notice that this was possible only if the cutting force components are proportional to each other; otherwise, more than one output signal is needed in order for the dynamometer to measure correct forces. 


\subsubsection{STRAIN RINGS}

Strain rings are structures used to measure force based on either the total deformation along an axis or strains of some points on the ring. Strain rings have a high ratio of sensitivity to stiffness and at the same time are very stable. In general, in machining processes, strain rings are used to measure the forces acting on the workpiece, as shown in Figure 5 [1].

To measure the forces $\mathrm{P}$ and $\mathrm{Q}$, the following two equations have been obtained [1]:

$$
\begin{aligned}
& \varepsilon_{1}= \pm \frac{1.09 \mathrm{Pr}}{E b t^{2}} \\
& \varepsilon_{2}= \pm \frac{2.18 Q r}{E b t^{2}}
\end{aligned}
$$

where $\varepsilon_{1}$ is the strain measured by the strain gages $\mathrm{T} 1$ and $\mathrm{C} 1$, and $\varepsilon_{2}$ is the strain measured by the strain gages $\mathrm{T} 2$ and $\mathrm{C} 2 ; \mathrm{P}$ and $\mathrm{Q}$ are the forces, $\mathrm{E}$ is Young's modulus of the ring material, $r$ is the mean radius of the ring, $b$ is the width of the ring, and $t$ the thickness of the ring's wall where the gages are installed. It is a characteristic of octagonal strain rings that the locations 1 and 2 , as shown in Figure 5, of the strain ring, with section 2 at about $45 \infty$ with respect to the horizontal axis, have no cross sensitivity. Consequently, gages at section 1 can be used to measure force $P$ independently of the value of force $Q$, and vice versa [1].

Requirements for the design of a strain ring are that the mean radius, $r$, must be as small as possible and the wall thickness, $t$, as large as possible, but still maintaining a good level of sensitivity [1].

Even though strain gage dynamometers have been widely utilized to measure cutting forces, some of them do not respond as fast as desired. Therefore, piezoelectric transducers have been used to do these tasks, like for example, in some cutting processes where the dynamic forces are needed to study the machinability of the material, the formation of the chip, chatter and wear of the tool [9].

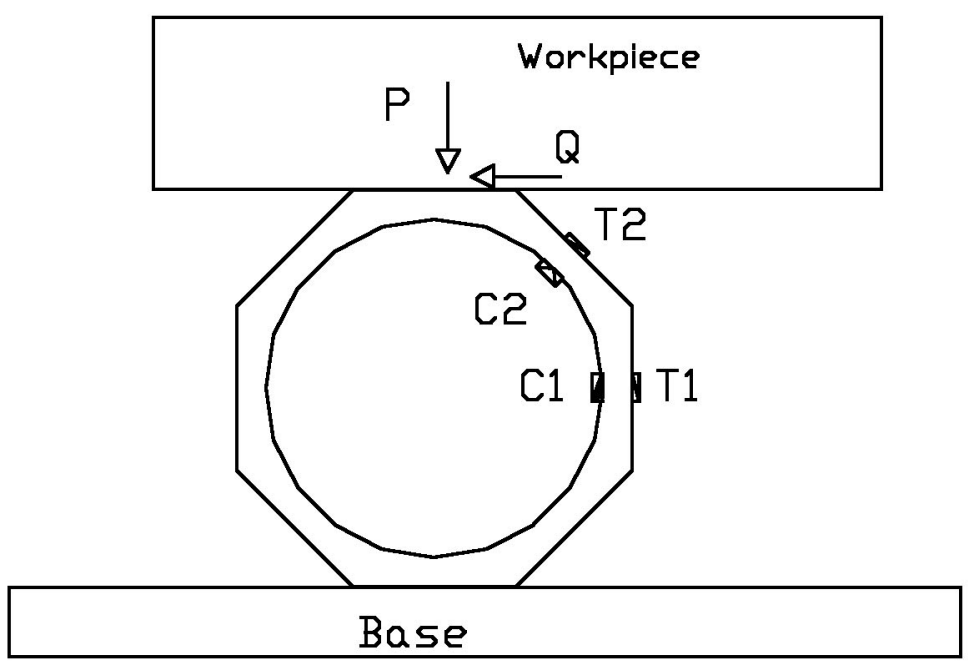

Figure 5. Strain Ring 


\subsection{PIEZOELECTRIC FORCE TRANSDUCERS}

Piezoelectric materials produce a voltage output when subjected to a force. These materials are very stiff and rigid, and have extremely high response, allowing to measure fast events. Piezoelectric transducer are ideal to measure dynamic forces, and cannot measure real static forces because the charge generated due to an static force decays and can only be measured temporarily. Therefore, highly insulated materials must be used for the cables and connectors to avoid losing the electric charge. Quartz is one of the best piezoelectric materials used to measure dynamic as well as static forces, because it has a very high insulation resistance, and complemented with charge amplifiers and signal conditioners, are able to measure static forces for minutes and maybe hours [8].

\subsubsection{QUARTZ SENSORS}

Dynamometers based on quartz 3-component force sensors started to be constructed in the early 70's, and consists of quartz discs cut along crystal planes particularly sensitive to shear, compression or tension forces. [3]. A stack of these quartz discs and electrodes are mounted in a pre-loaded housing to measure forces in three different mutually perpendicular directions. The stack of discs is pre-loaded to create the friction and contact required when transmitting shear forces. The quartz disks generate a voltage proportional to the force acting on their sensitivity axis [8].
Quartz sensor advantages [3, 8]:

- $\quad$ Absolute stability

- High rigidity

- High natural frequency

- $\quad$ Very low inherent cross-talk

- Linear with no hysteresis

- Almost constant sensitivity over a wide temperature range $\left(<500{ }^{\circ} \mathrm{C}\right)$.

- $\quad$ Extremely wide measuring dynamics

- $\quad$ Unlimited life

Applications of quartz sensors in machining processes [3]:

- $\quad$ Comparing cutting fluids

- $\quad$ Optimizing cutting parameters

- Comparing and selecting tools

- Determining tool wear, and causes of tool failure

Besides that, tasks that require wafer slicing, mirror finishing and precision grinding are some of the most demanding applications of these sensors [3].

Commercially, quartz transducers are classified as low or high impedance, and the difference is that the latest have external circuitry incorporated to amplify and convert electric charge to voltage [8]. Figure 6 presents the idea behind the piezo-electric sensors when telemetry equipment has been added to transmit the signal.

An advantage of piezo-electric sensors is that forces acting on the tool are measured directly by measuring voltage, and modular design of the dynamometer, with adapters, make them applicable to any machine tool [3]. 


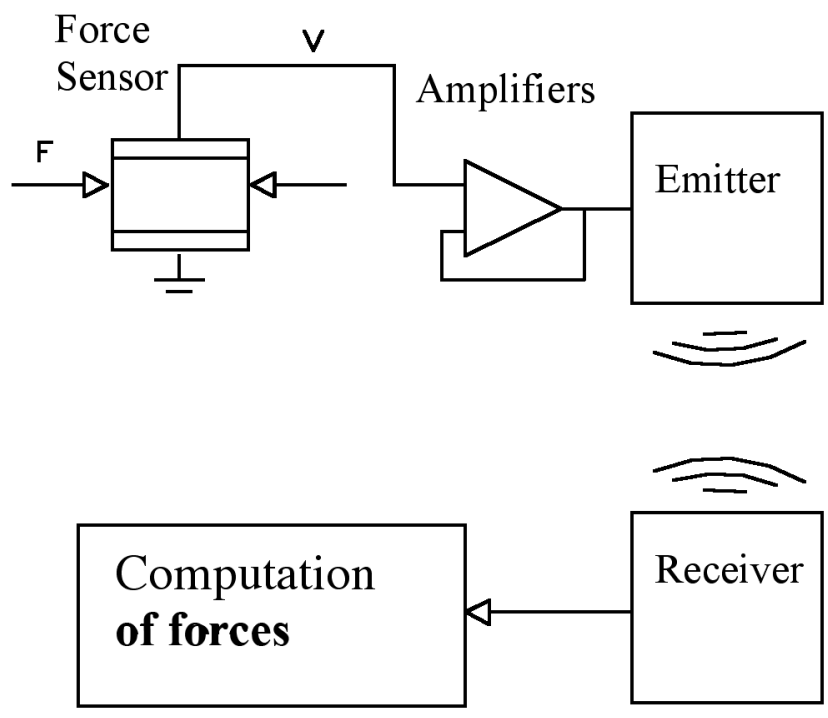

Figure 6. Piezo-electric sensor and signal conditioning

\subsubsection{POLYSILICON RESONANT TRANSDUCERS}

A polysilicon resonant transducer consists of a beam with such physical properties that its change in length, or strain, is converted to frequency, making possible its use as a force sensor [7]. A capacitive sensor or a piezo-resistive sensor is used to determine the vibration frequency of the resonator, which depends on the strain or deformation of the resonator structure; therefore, the vibration depends on the force to be measured. In Figure 7, there is a diagram of a resonator beam presenting two possible sensors to measure the frequency of vibration.
These type of devices could be built as micro-electro-mechanical systems (MEMS) which are very small devices and a recent technology that is in an experimental, developing, stage nowadays. However, it seems that in the future a great amount of transducers will be based on MEMS with polysilicon resonators. The development of new MEMS as force sensors will probably, in the near future, contribute to new ideas to incorporate force sensors in the tool shank, and to measure cutting forces in more efficient and useful way.

The whole structure of the resonant transducer could be considered somehow similar to a strain gage, with the difference that while strain gages suffer changes in electrical resistance, resonant transducers suffer changes in their frequency of 


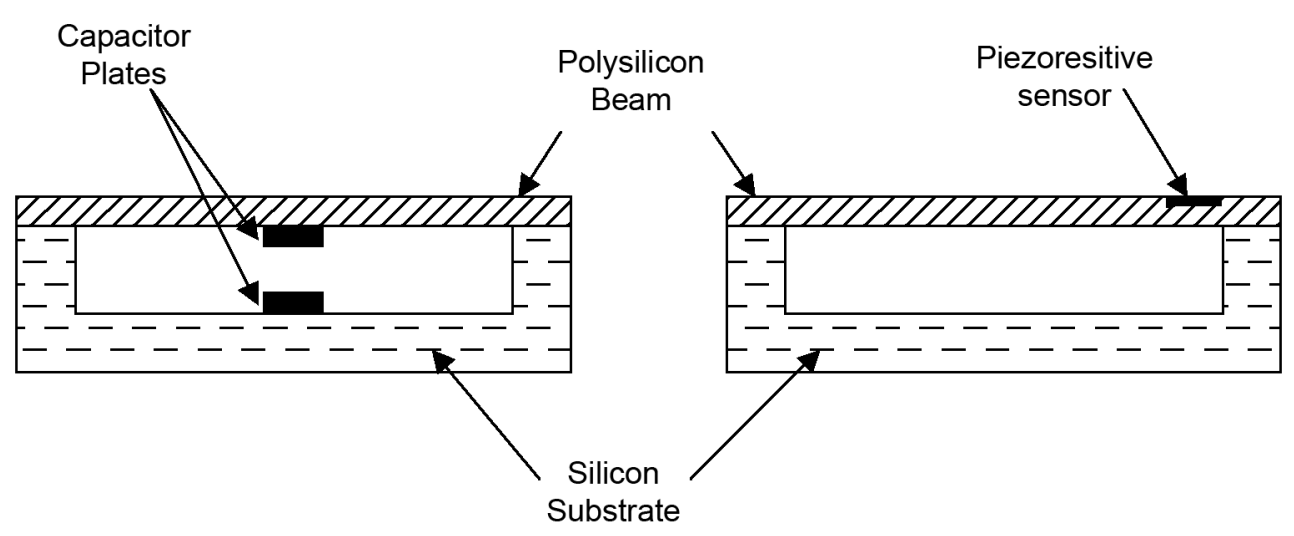

Figure 7. Resonant transducer with piezo-resistive sensors

\subsubsection{CAPACITIVE SENSORS}

A capacitive sensor consists of at least two plates that can hold an electric charge and which are separated by a dielectric element, as shown in Figure 8.

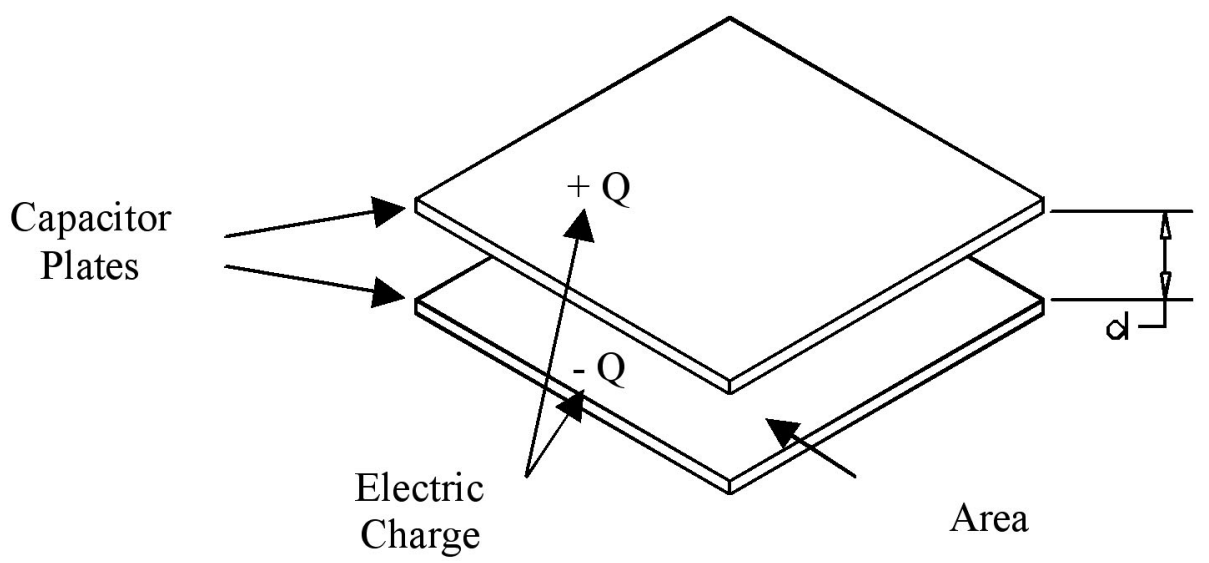

Figure 8. Capacitive Sensor

The capacitance is obtain with the next equation:

$$
C=\frac{\varepsilon_{0} \text { Area }}{d}
$$

where $\varepsilon_{\mathrm{O}}$ is the dielectric constant, or permittivity, of the material between the plates, $\varepsilon_{0}$ is 8,85 $\mathrm{x} 10^{-12} \mathrm{~F} / \mathrm{m}$ for free space or vacuum, and $\mathrm{d}$ is the distance between the plates.

Capacitance is usually measured indirectly by measuring voltage, and the configuration shown in Figure 9 is used for this purpose. 


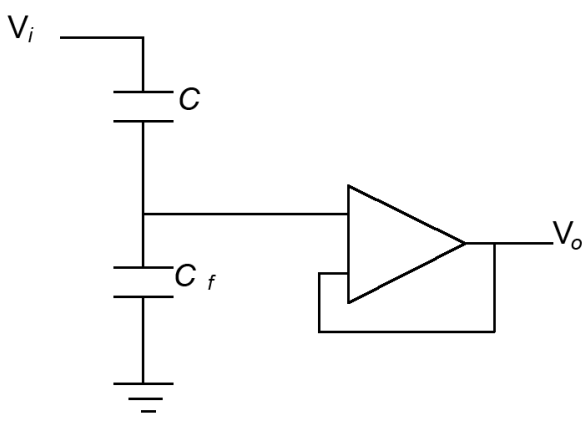

Figure 9. Capacitive Sensor Circuitry

Eq. (11) is the one used to measure the capacitance,

$V_{0}=\frac{C}{C+C_{f}} V_{i}$

where $\mathrm{C}_{\mathrm{f}}$ is a fixed known capacitor, of the same value as the capacitive sensor $\mathrm{C}$ when the plates are at the reference position, and $\mathrm{V}_{\mathrm{i}}$ and $\mathrm{V}_{\mathrm{O}}$ are the input and output voltages, respectively. A high impedance amplifier buffers the output voltage, between the capacitors $\mathrm{C}$ and $\mathrm{C}_{\mathrm{f}}$, in order to maintain the charge in the capacitor and also to avoid distorting the signal. Changes in output voltage are related to changes in capacitance as in Eq. (11). This way, the oscillation frequency of the beam in Figure 7 could be determined by analyzing the frequency of the output voltage of the capacitive sensor located under the beam. By measuring $\mathrm{V}_{\mathrm{o}}, \mathrm{C}$ is computed with Eq. (11) and after that the distance between the plates is obtained from Eq. (10).

Additionally, Figure 10 presents a possible structure to be used to install a capacitive sensor to measure forces. After dimensional analysis of this structure it was concluded that the following relationship applies [1]:

$\alpha \frac{E b^{2} a}{M_{Q}}=17\left(\frac{d}{a}\right)^{0.395}$

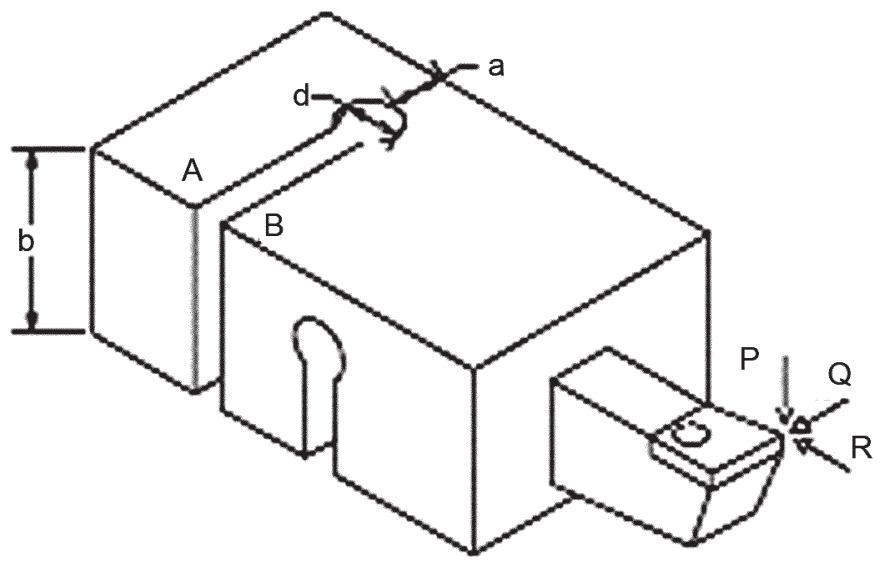

Figure 10. Dynamometer and Capacitive Sensors 


\section{CONCLUSION}

The purposes of this paper were to describe the principle of operation of force sensors most commonly used during machining processes and determine the trends of the technology in this field. It was obtained that cutting forces during machining are measured in most of the cases using dynamometers based on strain gages or piezoelectric transducer that contain quartz sensors. Measuring cutting forces during machining processes is considered as the most important and basic step to determine and control the conditions under which the machine, the tool, and the workpiece are performing. Force transducers, or sensors, are recently being designed and constructed as MEMS devices which use polysilicon beam resonator as sensors in order to relate the force that is being measured to the frequency of vibration of the resonator. Finally, the tendency of all recent and new dynamometers has been that the sensors are being installed on the tool holder or shank and the generated electrical signals, that carry information about the cutting forces, are being transferred by wireless technology.

\section{REFERENCES}

1. Shaw, Milton, 1 984, Metal Cutting Principles, Oxford, New York.
2. Kist, R., 1 986, Mechanical Problems in Measuring Force and Mass, H. Wieringa, Freiburg.

3. Dai, X.; Gautschi, G., 1 997, A New Generation of Cutting Force Dynamometers, Kistler Instruments AG, Switzerland.

4. Santochi, M.; Dini, G.: Tantussi, G., v46 n1 1 997, A sensor-integrated tool for cutting force monitoring. Manufacturing Technology.

5. Johnson, Thomas, Digital Load cell using small quartz sensors, Mechanical engineering Congress, 1994.

6. UW-MEMS. University of Wisconsin Madison, URL:

http://mems.engr.wics.edu/research/rft.html

7. URL: http://www.kistler.com/f-tech-forcetext.htm

8. Kim, Jeong-Du; Kim Dong-Sik, Nov. 23, 1 997, Development of a combined-type tool dynamometer with a piezo-film acceleromenter for an ultra-precision lathe. Journal of Materials Processing Technology, Korea.

9. Choudhurg, S.; Kishore, K. v40, n6, 2 000, Tool wear measurements in turning using force ratio. International Journal of Machine Tools and Manufacture. India. 
